\title{
Sperm Cryopreservation: Principles and Biology
}

\author{
Yashaswi Sharma, Mona Sharma* \\ Department of Reproductive Biology, All India Institute of Medical Sciences, New Delhi, India
}

Received: 28/07/2020

Accepted: 13/09/2020

Published: 20/09/2020

\begin{abstract}
Cryopreservation is a widely used method to preserve sperm prior to any cytotoxic therapy or testicular surgical intervention to be used later for assisted conception. Despite of multiple modifications in cryopreservation protocols, the yield of post-thaw good quality sperm has not been improved much. There is little data regarding factors affecting cryopreservation techniques and outcomes. Present review focuses on the basic biology of cryopreservation, its current protocols and effects on sperm proteomic and epigenomic modifications.
\end{abstract}

\section{Keywords: Cryopreservation, Spermatozoa, Fertility preservation, Cryoprotective agents, Cryobiology}

\section{Introduction}

Human semen cryopreservation history stretches back to over 200 years. The first attempt to cryopreserve semen was made by Lazaro Spallanzani in 1776 by cooling it in snow [1]. $\mathrm{He}$ is also known to have performed the first successful artificial insemination owing to the property of sperm recovering motility after freeze thaw. Further, in 1886, Mantegazza from Italy reported that human sperm survived cooling to $-17^{\circ} \mathrm{C}$ for more than 4 days [1]. This led to the idea of semen banking for the military personnel to safeguard the ability to procreate in the future. Thereafter, in an attempt to increase the time duration for which sperm could be frozen, various studies were conducted which came up with their own individualized techniques and protocols. However, only after about sixty years of time, significant scientific progress was made with the discovery of glycerol as an effective cryoprotectant for sperm by Polge [2].

Since then, glycerol has been regularly employed in animal sperm freezing, including that of humans and this is considered as a cornerstone in the field of fertility preservation. With the discovery of glycerol as sperm cryoprotective agent, there was a quick development in cryopreservation techniques that maintained sperm motility as well as its fertilizing ability post thaw. The earliest human offspring produced from cryopreserved spermatozoa were reported by Bunge and Sherman in 1953 [3]. Three pregnancies were reported on using the sperm which had been frozen in dry ice using glycerol [4]. After a decade to this, Sherman further showed how sperm could be stored for a longer duration if kept at $-196^{\circ} \mathrm{C}$ in liquid nitrogen (LN2). Cryopreservation of sperm has become a common practice today for artificial insemination of animals and human assisted reproductive technology. But it was an outcome of 20 years of continuous research before the first commercial sperm banks were created in the USA [4].

\section{Basic biology of sperm cryopreservation}

Cryopreservation refers to the maintenance of cellular life at subzero temperatures for a definite period of time [4]. Long term storage of cells including reproductive cells can be achieved if their metabolism is arrested and their cellular reactions retarded. The temperature of $\operatorname{LN} 2\left(-196^{\circ} \mathrm{C}\right)$ allows this since at such low temperatures no detectable biochemical activity is possible due to the lack of sufficient thermal energy. Moreover, the absence of liquid water limits all metabolic processes [5]. Thus, the storage in $\mathrm{LN} 2$ at $-196^{\circ} \mathrm{C}$ has become the standard approach for sperm freezing.

Sperm has small size with a large surface area. It is made up of considerable amount of water and has a high membrane fluidity as a result of the high sterol content of its membrane. The high amounts of polyunsaturated fatty acids (PUFA) present enables the sperm to survive rapid drops in temperature and makes it less susceptible to cold shock, possibly, since high cholesterol levels stabilize membranes during cooling. The lipid composition of the sperm plasma membrane is thus considered to be a major factor affecting the cryotolerance as well as the cold sensitivity of sperm [6].

The small size and large surface area of sperm makes them less susceptible to potential damage of cryopreservation [6]. However, the cellular survival is largely dependent on the cooling and thawing rate imposed during the cryopreservation process. The cooling rate must be such that it is slow enough to bypass intracellular ice crystal formation while fast enough not to cause immoderate cell dehydration followed by a rapid thawing rate. Solid crystalline structures called ice is formed on cooling water below its freezing point which is lighter and occupy a larger volume than liquid water. The expansion of these ice crystals during the solidification of water is the main factor that causes pressure and has a shearing effect on intracellular organelles resulting in appreciable damage [4].

In addition to this, as water solidifies, the solutes that were present in the water are excluded from the so formed ice, as a result the freezing point of the rest of the solution is lowered. The solution gets highly concentrated as the temperature drops leading to osmotic shock. There may be further damage to the cell as a result of disparity in osmolality gradient, causing release of reactive oxygen species which is highly detrimental to sperms [4].

The freeze-thaw process involves cooling and warming processes that may be lethal to the cells especially in the critical temperature range of $-10^{\circ} \mathrm{C}$ to $-60^{\circ} \mathrm{C}$ [7]. The sperm must undergo this temperature change two times during cooling and warming process. On warming, water re-enter the sperm and thus intracellular volume is restored, however, this invites the

*Corresponding authors: Mona Sharma, Associate Professor, Department of Reproductive Biology, All India Institute of Medical Sciences, New Delhi-110029, India. Contact No. 011-26594166; 9968147821. Email address: dr.mona18sharma@ gmail.com 
risk of intracellular ice crystal formation and subsequent cell injury. Hence, any cryopreservation protocol ensuring good sperm viability post thaw should target on controlled cell volume and avoid membrane damage by inhibiting ice crystal formation [4].

\section{Clinical applications of sperm cryopreservation}

Sperm cryopreservation is extensively used today as a routine technique in the clinical settings for fertility preservation, infertility treatment and establishment of donor banks. Men can choose to preserve their semen prior to any circumstances that may impair their fertility such as cancer patients before commencing treatment such as chemotherapy or radiotherapy, before undergoing surgery that may affect testicular function or lead to any form of sexual dysfunction, before onset of certain diseases and conditions such as an autoimmune disease or AIDS known to compromise fertility and in adult men who are seeking sterilization or sex reassignment surgery in case they want to have children in the future [7-11].

Moreover, sperm cryopreservation is also an important cornerstone in assisted reproduction technologies which primarily address infertility issues [12-14]. Patients with severe male infertility conditions like oligozoospermia or presence of intermittent motile spermatozoa are advised to freeze their sperms. Cryopreservation is also commonly used for surgical sperm recovery like percutaneous epididymal sperm aspiration (PESA), testicular sperm aspiration (TESA), or testicular sperm extraction (TESE) [15]. It also finds its applications in procedures like IUI, IVF and ICSI wherein sperm can be frozen from patients requiring assisted reproduction but face problems in sperm collection. Cryopreservation is also opted for patients with spinal cord injury coming for assisted ejaculation [4].

Artificial insemination is a way forward for an infertile man with no live spermatozoa or a single mother with desire to conceive. Stored semen from healthy and fertile donors may result in successful pregnancy in cases of recurrent miscarriages. Also, simple long-term storage of known quality donor semen is advised in many cases [11].

\section{Techniques with modified protocols}

Freezing techniques used in sperm cryopreservation involves two principal approaches- conventional freezing and vitrification. The conventional methods include slow freezing and fast freezing.

Slow freezing can be manual or automatic and as the name implies is done in phases. In a number of steps, the sperm is subjected to progressive cooling over a 2-3-hour period [11]. Manual method comprises of mixing the semen sample with an equal volume of cryoprotective agent (CPA) in a drop wise manner and then loading in straws or cryovials. It is further incubated at $40^{\circ} \mathrm{C}$ for 10 minutes and placed $15-20 \mathrm{~cm}$ above the level of LN2 for 15 minutes and completed by immersing in $\mathrm{LN} 2$ at $-196^{\circ} \mathrm{C}$ [4]. In the automated system, programmable freezers are used wherein software obtain cooling from $20^{\circ} \mathrm{C}$ to $-80^{\circ} \mathrm{C}$ at a rate of $1.5^{\circ} \mathrm{C} / \mathrm{min}$ and then at $6^{\circ} \mathrm{C} / \mathrm{min}$. Once the freezing is complete, the straws are plunged into LN2 at $196^{\circ} \mathrm{C}$. In vapor phase cooling, vapors of nitrogen that are constantly present around its tank is utilized for the cooling process. The vials are placed on cryocanes at certain specified heights above the liquid phase such that all vials are exposed to vapors equally. After a predetermined period of exposure, a cooling curve is attained [4]. Regardless of the method chosen it has been argued that conventional slow freezing leads to cell damage due to ice crystal formation, cell shrinkage, or osmotic changes [6].

In an attempt to overcome these drawbacks of slow conventional cooling, a remarkable fast approach to cryopreservation called vitrification has been proposed. The technique has already been widely explored and found its uses on embryos and oocytes and lately also for spermatozoa. [1621]. Different studies have described individual protocols using different carriers and methods for standardization of the vitrification procedure. But regardless of the protocol proposed, the principle behind the process is based on freezing of sperm by direct immersing it into LN2 which is at a freezing temperature of $-196^{\circ} \mathrm{C}$. [4]. This bypass the formation of intracellular ice crystals and thus the ensuing damage to the cell. In vitrification, water solidifies as an amorphous glass-like structure, and not as ice [7]. Vitrifying requires extremely high cooling rates $\left(>100,000{ }^{\circ} \mathrm{C} / \mathrm{min}\right)$ which can be obtained with different specifically designed packaging systems such as the open pulled straws, Flexipet denuding pipette, Cryotop, Cryoleaf, Cryotip and other carrier devices [4]. An alternative protocol involves the direct dropping of spermatozoa suspension in liquid nitrogen [7]. Rapid cooling rates have prevented cell shrinkage and osmotic cell damage. Since cryopreservation of sperm by vitrification avoids ice formation, requires minimal equipment, is time efficient and cost effective, it stands as a promising alternative to conventional methods of freezing [21].

\section{Use of Cryoprotectant}

Cryoprotectants are substances with high solubility used to protect biological tissue from cold shock during a freezing thawing process. They work by changing the solute concentration in the liquid phase by displacing water from intracellular to extracellular environment, lowering the freezing point of the solution. This limits water crystallization and thus cellular damage post-thaw/warm [4]. Depending upon their ability to cross the cell membrane, CPAs maybe of two types: permeating and non-permeating [7].

Permeating CPAs are compounds with relatively low molecular weight $(<100 \mathrm{~g} / \mathrm{mol})$ which freely cross membranes following osmolality gradient. Given to its ability to form hydrogen bonds with water, it prevents ice crystallization [4]. Dimethyl sulfoxide (DMSO), ethylene glycol, propylene glycol $(\mathrm{PROH})$ and glycerol are the most commonly used permeating cryoprotectants in sperm cryopreservation [22]. Almost all permeating CPAs for human sperm cryopreservation contain glycerol [22]. Glycerol, like other CPAs work by lowering the freezing point but also modify the lipid packing structure of cell membrane. Such induced changes bring about the reduction in the concentration of electrolyte in the unfrozen fraction [22]. Non- permeating cryoprotective agents on the other hand, are large molecules that remain extracellular. They work by creating osmolality gradient by drawing water from within the cell and are mostly used in combination with permeating CPAs to decrease cytotoxicity. Sucrose, raffinose and trehalose are the common non-permeating cryoprotectants used for sperm cryopreservation [23]. Usually, a combination of cryoprotectants are used in routine cryobiology. In an attempt to find the optimal additive that significantly improves cryosurvival rates, the use of glycine, citrate, egg yolk and zwitterions have also been explored [4]. Almost all the commonly used cryoprotective agents in sperm freezing contain glycerol as a thermal shock protecting agent, sugars for energy and optimization of $\mathrm{pH}$ and osmolality, egg yolk for 
plasma membrane fluidity and integrity and antibiotics for safeguarding against microorganisms [22].

Although the use of cryoprotectants is prescribed with the aim to maximize cell survival, it has also been shown that freezing methods which use permeable cryoprotectants may lead to cell injuries [7, 21-23]. The CPAs are osmotically active and thus their addition as well as removal during the freeze thaw process can cause lethal mechanical stress to the cells [22]. The most well studied cause for this is by the formation of intracellular and extracellular ice crystals. Moreover, chemical toxicity of the cryoprotectants on membrane and cellular components is also a concern. [21]. Permeable cryoprotectants are further hypothesized to have a negative influence on the genetic apparatus of spermatozoa [23]. Hence, some recent studies have been conducted with the aim to find the possibility of sperm cell freezing without the use of cryoprotectants [24, 25].

\section{Changes in sperm parameters during cryopreservation}

Although it has been well established that sperm are less susceptible to freezing damages than other cell types, significant structural and functional damage to sperm have been reported post cryopreservation. Thermal shock due to drastic changes in temperature, osmotic shock, dehydration of cells and formation of ice crystals are the most commonly reported mechanisms by which cryodamage to the cell is incurred [4]. The inevitable change involves the significant reduction in motility mostly due to membrane changes and acrosome degeneration among the others. A decrease in spermatozoa kinetics after thawing has been reported consistently in many studies [26-29].

Damage is incurred due to the peroxidation of the fatty acids present in the sperm plasma membrane. The lipid oxidation results in the inhibition of oxidative phosphorylation by loss of intracellular enzymes. The ultrastructure of the sperm mitochondria and plasma membrane is particularly susceptible to freezing damage as shown by studies employing electron microscopes [4]. The widespread damage to the cells is mostly either due to alteration in mitochondrial functions due to significant destruction of mitochondrial membrane or an alteration in the fluidity of the membrane leading to the liberation of reactive oxygen species (ROS) [30]. ROS have been well recognized as cell destructive agents most commonly causing single- or double-strand DNA breakage. They are also responsible for inducing apoptotic pathways in cell under low activity of antioxidant enzymes which subsequently leads to decreased sperm viability [31].

The integrity of cell membrane is crucial for sustained functioning of the cell post freeze thaw. However, low temperatures have been suggested to alter the membrane proteins and carbohydrate structures either due to osmolality changes or intracellular ice crystal formation [7]. This may in turn impair ion transport and metabolism leading to cell disruption and consequently in reduction of sperm viability and fertilization capability [4].

Another parameter affected by freezing is sperm morphology which mostly results due to the deformation in membrane structure due to dysregulated liquid influx $[2,7,27$, 29]. It has also been shown that freeze preservation of sperm is followed by ultrastructural changes [11]. The most conspicuous of changes are reported in the acrosomal region wherein limiting membranes are found to be more wrinkled and less clearly defined [30]. There is partial or complete disintegration of the acrosomal membrane with depletion of acrosomal content, swelling and thinning of the area between the cell membrane and the acrosome [7]. Changes in the middle piece and axial filament complex are also observed. Scanning electron microscopy studies report a significant increase of head and tail defects in post freeze sample where coiled and looped tails of sperms is the most common finding [32].

Although the detrimental effects of freeze-thaw cycles on various sperm parameters and fertilization capacity is well elucidated, there are two opposite thoughts regarding the DNA damage imposed by such procedures. A set of studies suggest that cryopreservation of sperm exert a negative impact on DNA integrity and causes its fragmentation due to oxidative stress caused by ROS [33-36]. The second line of thought believes that sperm DNA integrity is not affected by freezing and thawing process [37-39].

Studies carried out to assess the damage on DNA post cryopreservation utilize a battery of tests such as TUNEL, SCSA, SCD, Comet neutral or Comet alkaline which might help answer the discrepancies in findings of these studies. Also, disparity in the cryo-resistance of each different sample, protocol followed during the cryopreservation process, and the method of choice to assess DNA integrity may account for the non-consensus between studies.

\section{Other modifications in post-thaw sperm (proteomic, transcriptomic/ epigenomic)}

The freeze thaw process is quite elaborative as it not only alters the sperm parameters but also the genes, mRNA stability, protein expression and the epigenetic content of the spermatozoa. In post thaw sperm samples, protein degradation and phosphorylation have been reported [6]. Protein phosphorylation has also been linked to capacitation like changes in sperm which is believed to shorten its lifespan. Protein expression differences have been reported in multiple proteins of boar sperm post thaw compared to its pre freeze samples [40]. Some proteins have been shown to increase in frozen thawed sperm such as AKAP3, superoxide dismutase 1 (SOD1), TPI1 and ODF2 proteins [41]. The expression levels of heat shock protein 90 (HSP90) having its direct role in motility of sperm is found to be significantly decreased after cryopreservation [42]. Significant changes in proteins related to motility, viability and acrosomal integrity of spermatozoa, such as mitochondrial aconitase hydratase (ACO2), alphaenolase (ENO1), OXCT1, tektin1 (TEKT1), acrosome membrane-associated protein 3 (SPACA3), vimentin, etc. has been shown to change after freezing $[43,44]$.

Cryopreservation has also been suggested to negatively influence the development of early embryo by increasing the risk of loss of important transcripts and mRNA-protein interactions in sperms $[44,45]$. The correlation of sperm mRNA and early embryo development is shown for both humans and animals like pig. A number of transcripts having definite roles in human sperm fertility such as PRM1, PRM2, PEG1/MEST, eif2S1 and ADD1 have been reported to have decreased expression post cryopreservation [44]. Since damage to the sperm genetic material do not always restrict fertilization capability, mutations and defects may be evident only after sufficient growth of the embryo or on the development of fetus [46].

Some studies explain the possible cryogenic epigenetic modifications in the sperm. Although most of these studies have been conducted on animal sperm samples, they report alterations in histone H1-DNA binding proteins, protein-DNA 
disulphide bonds and high methylation in the vasa and cxcr4b promoters in boar and zebrafish respectively [47]. A particular study on DNA methylation pattern of human sperm genes, however, report no changes pre and post freezing [48].

\section{Solution to Cryoinjuries}

Cryoinjuries to sperm can be limited during the cryopreservation process by using certain substances that are known to have cryoprotective properties. Cryoprotectants, are one such substance which depending upon their size and permeability exert their cryoprotective effect by decreasing the freezing point of intracellular and extracellular water [22]. They protect both cytoplasmic components as well as sperm membranes by forming a protective layer around them. Other additives that are reported to protect sperm against damage include antioxidants which primarily work to neutralize ROS generated during the cryopreservation process and thus improve post-thaw sperm function. The commonly employed antioxidants include vitamin $\mathrm{E}$, vitamin $\mathrm{C}$, catalase, 1-carnitine, biotin, butylated hydroxytoluene, taurine, hyaluronic acid, resveratrol, honey, and nerve growth factor [49-53]. Antifreeze proteins and glycoproteins are another group of cryoprotective supplements that can be employed in sperm freezing that help to maintain the plasma membrane integrity by stabilizing the phospholipids and unsaturated fatty acids [50]. The use of such proteins is also reported to act by decreasing the freezing point and inhibiting ice crystal formation [22]. Although the beneficial role of these antifreeze compounds has not been well established for human sperm cryopreservation, promising results have been shown in animals. Other additives that help to reduce cryoinjuries include fatty acids, animal serum, nanoparticles or plant essential oils which are added to the freezing media to protect sperm against damage [6].

Besides the use of supplements, other noble strategies to reduce cryodamage in sperm include the use of magnetized water as one of the components of freezing media which allow only small ice crystals to form on freezing, stress preconditioning of spermatozoa before cryopreservation to induce adaptation to stress and the use of low-level laser irradiation to affect the sperm's mitochondrial respiratory chain such that ATP production is increased while ROS decreased $[54,55,56]$.

\section{Use of sperm cryopreservation with or without semen}

The somatic cells contain antioxidants in cytoplasm which protect them from oxidative insult. During maturation however, sperm lose a large portion of their cytoplasm and as a result fall short on enzymes and repairing mechanism for oxidative damage leaving them susceptible to freeze-thaw damage by ROS [57]. However, it has been shown that the seminal plasma has an anti ROS activity against $\mathrm{O} 2$ and $\mathrm{H} 2 \mathrm{O} 2$ by virtue of its enzymes: superoxide dismutase, glutathione reductase/peroxidase and catalase [58]. It has also been proposed that the lipoproteins are responsible for stabilizing the plasma membrane by maintaining the optimal lipid composition. It has been observed that cryostability of the cellular and plasma membrane of sperm is increased during cryopreservation [57]. Studies on such antioxidant properties of human, rat and mouse seminal plasma have been described and proven $[59,60]$.

Hence, cryopreserving sperm with semen or seminal plasma has been supported by a number of studies for the above reasons. These ideas are further endorsed by studies which involve stripping sperm from the protective seminal plasma and demonstrating the adverse effect of ROS on cell organelles including cell DNA [57]. Further, such damage has been shown to be successfully avoided by supplementing media with antioxidants.

However, there are studies suggesting that seminal leukocytes and damaged sperm in semen produce ROS which damage ejaculated sperm upon prolonged exposure leading to reduction in motility and viability of sperm [61, 62]. In accordance to this belief, it has been suggested that ejaculated sperm should be separated from the seminal environment as soon as possible.

\section{Factors affecting outcome of freeze thaw cycle}

The outcome of a freeze thaw cycle of sperm is dependent on multiple factors. The type of cell undergoing freezing, its size and maturity, composition of the intracellular lipids, amount of water present, function and morphology all determine the cryosurvival potency of the frozen cell irrespective of the freezing method employed. Resistance to cryoinjuries is also dependent on sperm size, shape and lipid composition as it varies between different animals and between different species of the same animal [6]. The difference in lipid composition in terms of fatty acid profile and omega-3/ omega6 ratio also decide their cryotolerance level [4].

The pre-thaw properties of sperm are also likely to have an effect on the post-thaw quality of sperm. For instance, sperm with abnormal motility traits such as asthenozoospermia and oligoasthenozoospermia are found to be more susceptible to cryodamage. Studies have concluded that sub-normal semen samples do not withstand freezing and the yield of the sperm post-freezing bears a linear relationship with the pre-freeze quality $[63,64]$. Also, the period of abstinence of sperm donors has been shown to affect the cryosurvival rate of post-thaw sperm. The subnormal samples are more prone to DNA damage induced during freeze-thawing and has a higher incidence of irregular chromatin organization and decreased resistance to thermal denaturation as compared to normal sperm samples [65].

Besides these factors, the method of cryopreservation employed (slow freezing or vitrification), the use or disuse of cryoprotectant, the type (permeable or non-permeable or mix of both), concentration of cryoprotectant or other additives used and the protocol followed significantly impacts the outcome of cryopreservation [4]. Among the other variation in protocols, the cooling and warming rate employed and the temperature at which the sample is plunged into LN2 and the subsequent sample storage temperature has a significant effect on the outcome of the freeze thaw cycle.

\section{Future of sperm cryopreservation}

The field of cryobiology is in a rapid state of flux and has found its applications in fertility medicine. Since it is a cuttingedge technology, it has a great potential for further development. It is also utmost important to assess the extent of sperm cryopreservation being used clinically for assisted conception. Although sperm cryopreservation has been used for preserving fertility in adult men, it further needs to find its application in fertility preservation in boys preceding puberty. The germ stem cells of the seminiferous epithelium in the testes called spermatogonial stem cells and even testicular tissue stand as promising alternatives to ejaculated spermatozoa which may be cryopreserved to be retrieved later $[66,67,68]$. Also, sperm vitrification, which stands as a promising 
invaluable technology in the field of reproductive medicine needs further studying. As of now only protocols describing the vitrification of very small volume of semen sample are reported; upto about $10 \mu \mathrm{L}$ with isolation from $\mathrm{LN} 2$ and upto $30 \mu \mathrm{L}$ with direct plunging into LN2 [24, 38]. Although use of vitrification has been encouraged, still some studies have shown detrimental effects on post-thaw quality of gametes [69, 70]. Shortcomings need to be studied and may be reflected in non-uniformity of the vitrification regime.

\section{Conclusions}

A thorough understanding of current concepts of sperm cryopreservation influenced by epigenomic and proteomic modifications will enable efficient clinical application in assisted reproduction. There is a serious need for the vitrification process to be standardized owing to its promising advantages over the conventional methods.

\section{Ethical issue}

Authors are aware of, and comply with, best practice in publication ethics specifically with regard to authorship (avoidance of guest authorship), dual submission, manipulation of figures, competing interests and compliance with policies on research ethics. Authors adhere to publication requirements that submitted work is original and has not been published elsewhere in any language.

\section{Competing interests}

The authors declare that there is no conflict of interest that would prejudice the impartiality of this scientific work.

\section{Authors' contribution}

All authors of this study have a complete contribution for data collection, data analyses and manuscript writing.

\section{References}

1. Royere, D., Barthelemy, C., Hamamah, S., and Lansac, J. Cryopreservation of sperma spermatozoa: A 1996 review. Hum. Reprod 1996; 2: 553-559

2. ErdemÖztürk A, NumanBucak M, Bodu M, Başpınar N, Çelik İ, Shu Z, Keskin N., and Dayong G.Cryobiology and Cryopreservation of Sperm, Cryopreservation - Current Advances and Evaluations, Marian Quain, IntechOpen, 2019. Available from: https://www.intechopen.com/books/cryopreservationcurrent-advances-andevaluations/cryobiology-andcryopreservation-of-sperm

3. Natarajamani S. Cryopreservation of Human Semen: Male infertility: A clinical approach.2016: $207-219$

4. Indian Fertility Society. Semen Freezing Past, Present \& Future. 2017, Available from https://indianfertilitysociety.org/pdf/nexus_4_Final_29.01.17_8.1 5pm.pdf (Accessed June 3, 2020).

5. Rios A, Botella I. Causes and Impact of CryopreservationAssociated Damage on Different Parameters of Human Spermatozoa and its Clinical Impact. EMJ 2019; 5(1):100-109.

6. Hezavehei M, Sharafi M, Kouchesfahani HM, et al. Sperm cryopreservation: A review on current molecular cryobiology and advanced approaches. Reprod Biomed Online. 2018; 37 (3):327339.

7. Isachenko E, Rahimi G, Mallmann P et al, Novel Approaches to the Cryopreservation of Human Spermatozoa: History and Development of the Spermatozoa Vitrification Technology. JRSCB. 2011; 2 (2):128-145.

8. Williams DH. Sperm banking and the cancer patient. TherAdv Urol. 2010; 2(1):19-34.

9. Ajala T, Rafi J, Larsen-Disney P. and Howell R. Fertility preservation for cancer patients: a review. ObstetGynecol Int. 2010; 2010: 160386.
10. Multidisciplinary Working Group convened by the British Fertility Society. A strategy for fertility services for survivors of childhood cancer. Hum Fertil (Camb). 2003;6 (2):A1-A39.

11. Di Santo M, Tarozzi N, Nadalini M, and Borini A. Human Sperm Cryopreservation: Update on Techniques, Effect on DNA Integrity, and Implications for ART. Adv Urol. 2012; 2012:1-12.

12. Poongothai J. Etiology, investigation and treatment of Human men's infertility. J. Infertil. Reprod. Biol. 2013; 1(2): 31-36.

13. Sheykhhasan M., Ghias M. Semen quality and age-dependent changes among male participants with normal sperm count in Qom, Iran. . J. Infertil. Reprod. Biol.2016; 4(2):35-39.

14. Eftekhar M., Pourmasumi S., and Razi MH. Efficacy of rescue ICSI after total fertilization failure in conventional IVF. J. Infertil. Reprod. Biol.2013; 1(2):58-62.

15. Gangrade BK. Cryopreservation of testicular and epididymal sperm: techniques and clinical outcomes of assisted conception. Clinics (Sao Paulo). 2013;68 Suppl 1(Suppl 1):131140.

16. Konc J, Kanyó K, Kriston R, et al. Cryopreservation of embryos and oocytes in human assisted reproduction. Biomed Res Int. 2014; 2014:307268 (1-9).

17. Wilmut I. The low temperature preservation of mammalian embryos. J ReprodFertil. 1972;31 (3):513-514

18. Le MT, Nguyen TTT, Nguyen TT, et al. Cryopreservation of human spermatozoa by vitrification versus conventional rapid freezing: Effects on motility, viability, morphology and cellular defects. Eur J ObstetGynecolReprod Biol. 2019;234:14-20.

19. Khalili M, Adib M and Ramezani M. Cryopreservation of human spermatozoa by vitrification: impacts on sperm parameters and apoptosis. FertilSteril. 2010;94(4):S108.

20. Berkovitz A, Miller N, Silberman M, Belenky M, Itsykson P. A novel solution for freezing small numbers of spermatozoa using a sperm vitrification device. Hum Reprod. 2018;33(11):1975-1983.

21. He X, Park EY, Fowler A, Yarmush ML, Toner M. Vitrification by ultra-fast cooling at a low concentration of cryoprotectants in a quartz micro-capillary: a study using murine embryonic stem cells. Cryobiology. 2008;56 (3):223-232.

22. Sieme $\mathrm{H}$, Oldenhof $\mathrm{H}$, Wolkers WF. Mode of action of cryoprotectants for sperm preservation. AnimReprod Sci. 2016;169:2-5

23. Sztein J, Noble K, Farley J, Mobraaten L. Comparison of Permeating andNonpermeating Cryoprotectants for Mouse Sperm Cryopreservation. Cryobiology. 2001;42(1):28-39.

24. Nawroth F, Isachenko V, Dessole S, Isachenko E. Successful cryopreservation of human spermatozoa by direct plunging into liquid nitrogen (vitrification) without cryoprotectants. FertilSteril. 2002;78:S129.

25. Isachenko V, Maettner R, Petrunkina AM, et al. Vitrification of human ICSI/IVF spermatozoa without cryoprotectants: new capillary technology. J Androl. 2012;33(3):462-468.

26. Keel B and Black J. Reduced Motility Longevity in Thawed Human Spermatozoa. Archives of Andrology. 1980; 4 (3):213215 .

27. Łukaszewicz E and Kruszyński W. Evaluation of fresh and frozenthawed semen of individual ganders by assessment of spermatozoa motility and morphology. Theriogenology. 2003;59(7):16271640.

28. Oberoi B, Kumar S andTalwar P. Study of human sperm motility post cryopreservation. Med J Armed Forces India. 2014;70 (4):349-353.

29. Darsini N, Hamidah B, Suyono S, et.al. Human Sperm Motility, Viability, and Morphology Decreased after Cryopreservation. Folia MedicaIndonesiana. 2019;55(3):198.

30. Barthelemy C, Royere D, Hammahah S, Lebos C, Tharanne M, Lansac J. Ultrastructural Changes in Membranes and Acrosome of Human Sperm During Cryopreservation. Archives of Andrology. 1990;25(1):29-40

31. Chris W. and Ford L. Reactive oxygen species and sperm. Hum Fertil. 2001; 4(2): 77-78.

32. Ozkavukcu S, Erdemli E, Isik A, et al. Effects of cryopreservation on sperm parameters and ultrastructural morphology of human spermatozoa. J Assist Reprod Genet. 2008;25(8):403-411. 
33. Chohan K, Griffin J, Carrell D. Evaluation of chromatin integrity in human sperm using acridine orange staining with different fixatives and after cryopreservation. Andrologia. 2004;36(5):321326

34. Hosseini A, Khalili M, Talebi A, Agha-Rahimi A, GhasemiEsmailabad S, Woodward B et al. Cryopreservation of Low Number of Human Spermatozoa; Which is Better: Vapor Phase or Direct Submerging in Liquid Nitrogen?. Hum Fertil. 2018; 22(2):126-132.

35. Spanò M, Cordelli E, Leter G, et al. Nuclear chromatin variations in human spermatozoa undergoing swim-up and cryopreservation evaluated by the flow cytometric sperm chromatin structure assay. Mol Hum Reprod. 1999;5(1):29-37.

36. de Paula T, Bertolla R, Spaine D, Cunha M, Schor N, Cedenho A. Effect of cryopreservation on sperm apoptotic deoxyribonucleic acid fragmentation in patients with oligozoospermia. FertilSteril. 2006; 86 (3):597-600.

37. Lusignan M, Li X, Herrero B, et al. Effects of different cryopreservation methods on DNA integrity and sperm chromatin quality in men. J Androl y. 2018;6(6):829-835.

38. Isachenko $\mathrm{E}$, Isachenko $\mathrm{V}$, Katkov II, et al. DNA integrity and motility of human spermatozoa after standard slow freezing versus cryoprotectant-free vitrification. Hum Reprod. 2004;19(4):932939

39. Morshedi M, Duru N, Oehninger S. Cryopreservation-Thawing Does Not Impair DNA Integrity but Induces Plasma Membrane Translocation of Phosphatidylserine in Fractionated Human Spermatozoa. FertilSteril. 2000;74 (3):S244-S245

40. Chen X, Zhu H, Hu C, et al. Identification of differentially expressed proteins in fresh and frozen-thawed boar spermatozoa by iTRAQ-coupled 2D LC-MS/MS. Reproduction. 2014;147(3):321-330.

41. Bogle OA, Kumar K, Attardo-Parrinello C, et al. Identification of protein changes in human spermatozoa throughout the cryopreservation process. Andrology. 2017;5(1):10-22.

42. Huang SY, Pribenszky C, Kuo YH, et al. Hydrostatic pressure pretreatment affects the protein profile of boar sperm before and after freezing-thawing. AnimReprod Sci. 2009; 112 (1-2):136-149.

43. Wang S, Wang W, Xu Y, et al. Proteomic characteristics of human sperm cryopreservation. Proteomics. 2014; 14(2-3):298-310.

44. Valcarce DG, Cartón-García F, Herráez MP, Robles V. Effect of cryopreservation on human sperm messenger RNAs crucial for fertilization and early embryo development. Cryobiology. 2013;67(1):84-90.

45. Valcarce DG, Cartón-García F, Riesco MF, Herráez MP, Robles V. Analysis of DNA damage after human sperm cryopreservation in genes crucial for fertilization and early embryo development. Andrology. 2013; 1(5):723-730.

46. Twigg JP, Irvine DS, Aitken RJ. Oxidative damage to DNA in human spermatozoa does not preclude pronucleus formation at intracytoplasmic sperm injection. Hum Reprod 1998;13:1864-71

47. Flores E, Ramió-Lluch L, Bucci D, et al. Freezing-thawing induces alterations in histone H1-DNA binding and the breaking of protein-DNA disulfide bonds in boar sperm. Theriogenology. 2011;76(8):1450-1464.

48. Kläver R, Bleiziffer A, Redmann $\mathrm{K}$, et al. Routine cryopreservation of spermatozoa is safe--evidence from the DNA methylation pattern of nine spermatozoa genes. J Assist Reprod Genet. 2012;29(9):943-950.

49. Fattah A, Sharafi M, MasoudiR,et al. L-Carnitine in rooster semen cryopreservation: Flow cytometric, biochemical and motion findings for frozen-thawed sperm. Cryobiology. 2017;74:148-153.

50. Najafi A, Najafi M, Zanganeh Z, et al. Cryopreservation of Ram Semen in Extenders Containing Soybean Lecithin as Cryoprotectant and Hyaluronic Acid as Antioxidant. Reproduction in Domestic Animals. 2014;49(6):934-940.

51. Saeednia S, ShabaniNashtaei M, Bahadoran H, et al. Effect of nerve growth factor on sperm quality in asthenozoosprmic men during cryopreservation. ReprodBiolEndocrinol. 2016;14(1):29.

52. ShabaniNashtaei M, Amidi F, Sedighi Gilani M, et al. Protective features of resveratrol on human spermatozoa cryopreservation may be mediated through 5' AMP-activated protein kinase activation. Andrology. 2016;5(2):313-326.
53. Partyka A, Rodak O, Bajzert J, Kochan J and Niżański W. The Effect of L-Carnitine, Hypotaurine, and Taurine Supplementation on the Quality of Cryopreserved Chicken Semen. Biomed Res Int. 2017;2017:7279341

54. Feyzi S, Sharafi M, Rahimi S. Stress preconditioning of rooster semen before cryopreservation improves fertility potential of thawed sperm. Poult Sci. 2018;97(7):2582-2590.

55. Cai R, Yang H, He J, Zhu W. The effects of magnetic fields on water molecular hydrogen bonds. J MolStruct. 2009;938(1-3):1519.

56. Iaffaldano N, Rosato MP, Paventi G, et al. The irradiation of rabbit sperm cells with $\mathrm{He}-\mathrm{Ne}$ laser prevents their in vitro liquid storage dependent damage. AnimReprod Sci. 2010; 119 (1-2):123-129.

57. Donnelly E, McClure N, Lewis S. Cryopreservation of human semen and prepared sperm: effects on motility parameters and DNA integrity. FertilSteril. 2001; 76 (5):892-900.

58. Moore A, Squires E, Graham J. Effect of seminal plasma on the cryopreservation of equine spermatozoa. Theriogenology. 2005; 63 (9):2372-2381

59. Tavilani H, Goodarzi MT, Doosti M, et al. Relationship between seminal antioxidant enzymes and the phospholipid and fatty acid composition of spermatozoa. Reprod Biomed Online. 2008; 16 (5):649-656. doi:10.1016/s1472-6483(10)60478-6

60. Peeker R, Abramsson L, Marklund SL. Superoxide dismutase isoenzymes in human seminal plasma and spermatozoa. Mol Hum Reprod. 1997;3(12):1061-1066.

61. Moein, M., Dehghani, V., Tabibnejad, N. and Vahidi, S. Reactive Oxygen Species (ROS) level in seminal plasma of infertile men and healthy donors. Iran J Reprod Med.2007; 5(2): 51-55.

62. Mupfiga C, Fisher D, Kruger T, Henkel R. The relationship between seminal leukocytes, oxidative status in the ejaculate, and apoptotic markers in human spermatozoa. SystBiolReprod Med. 2013; 59 (6):304-311.

63. Rath SK, Tarneja P, Singh M. Effect of Cryopreservation on Semen Sample. Med J Armed Forces India. 2004; 60 (1):42-44.

64. Verza S Jr, Feijo CM, Esteves SC. Resistance of human spermatozoa to cryoinjury in repeated cycles of thawrefreezing. IntBraz J Urol. 2009; 35 (5):581-591.

65. Kalthur G, Adiga SK, Upadhya D, Rao S, Kumar P. Effect of cryopreservation on sperm DNA integrity in patients with teratospermia. FertilSteril. 2008; 89 (6):1723-1727.

66. Sinha N, Whelan EC, Brinster RL. Isolation, Cryopreservation, and Transplantation of Spermatogonial Stem Cells. Methods Mol Biol. 2019; 2005: 205-220.

67. Aliakbari F, Yazdekhasti H, Abbasi M, HajianMonfared M, Baazm M. Advances in cryopreservation of spermatogonial stem cells and restoration of male fertility. Microsc Res Tech. 2016; 79 (2):122-129.

68. Vermeulen M, Giudice MG, Del Vento F, Wyns C. Role of stem cells in fertility preservation: current insights. Stem Cells Cloning. 2019; 12: 27-48

69. Taherzadeh S, Khalili MA, Agha-Rahimi A, Anbari F, Ghazali S, Macchiarelli G. Vitrification Increased Vacuolization of Human Spematozoa: Application of MSOME Technology. J Reprod Infertil. 2017; 18 (2):225-230.

70. Amoushahi M, Salehnia M, Mowla SJ. Vitrification of Mouse MII Oocyte Decreases the Mitochondrial DNA Copy Number, TFAM Gene Expression and Mitochondrial Enzyme Activity. J Reprod Infertil. 2017; 18 (4):343-351. 